\title{
BMJ Open Impact of COVID-19 and other pandemics and epidemics on people with pre-existing mental disorders: a systematic review protocol and suggestions for clinical care
}

\author{
Anjali Sergeant (D) , ${ }^{1}$ Emma Alaine van Reekum, ${ }^{2}$ Nitika Sanger (D),${ }^{3}$ \\ Alexander Dufort, ${ }^{2}$ Tea Rosic (1) , ${ }^{2}$ Stephanie Sanger, ${ }^{4}$ Sandra Lubert, ${ }^{5}$ \\ Lawrence Mbuagbaw (1) , ${ }^{6}$ Lehana Thabane, ${ }^{6}$ Zainab Samaan (1) ${ }^{2}$
}

To cite: Sergeant $A$, van Reekum EA, Sanger N, et al. Impact of COVID-19 and other pandemics and epidemics on people with pre-existing mental disorders: a systematic review protocol and suggestions for clinical care. BMJ Open 2020;10:e040229. doi:10.1136/ bmjopen-2020-040229

- Prepublication history for this paper is available online. To view these files, please visit the journal online (http://dx.doi. org/10.1136/bmjopen-2020040229).

AS and EAvR contributed equally.

Received 12 May 2020 Revised 28 July 2020 Accepted 06 August 2020

Check for updates

(c) Author(s) (or their employer(s)) 2020. Re-use permitted under CC BY-NC. No commercial re-use. See rights and permissions. Published by BMJ.

For numbered affiliations see end of article.

Correspondence to

Dr Zainab Samaan;

samaanz@mcmaster.ca

\section{ABSTRACT}

Introduction The current COVID-19 pandemic has resulted in high rates of infection and death, as well as widespread social disruption and a reduction in access to healthcare services and support. There is growing concern over how the pandemic, as well as measures put in place to curb the pandemic, will impact people with mental disorders. We aim to study the effect of pandemics and epidemics on mental health outcomes for people with premorbid mental disorders.

Methods and analysis With our predefined search strategy, we will search five databases for studies reporting on mental health outcomes in people with pre-existing mental disorders during pandemic and epidemic settings. Search dates are planned as follows: 5 May 2020 and 23 July 2020. The following databases will be searched: MEDLINE/PubMed, CINAHL, PsycINFO, MedRxiv and EMBASE. Data will be screened and extracted in duplicate by two independent reviewers. Studies involving non-clinical populations or patients diagnosed with a mental disorder during a pandemic/epidemic will be excluded We will include data collected from all pandemics and epidemics throughout history, including the present COVID-19 pandemic. If possible, study findings will be combined in meta-analyses, and subgroup analyses will be performed. We hope that this review will shed light on the impact of pandemics and epidemics on those with pre-existing mental disorders. Knowledge generated may inform future intervention studies as well as healthcare policies. Given the potential implications of the current pandemic measures (ie, disruption of healthcare services) on mental health, we will also compile a list of existing mental health resources.

Ethics and dissemination No ethical approval is required for this protocol and proposed systematic review as we will only use data from previously published papers that have themselves received ethics clearance and used proper informed consent procedures.
Strengths and limitations of this study

A major strength is that we consulted people with lived experience during all phases of this proposed project; by giving patients a strong voice, we can ensure that this research is important and meaningful to those who are most at stake.

- Another strength is our proposed timeline: we expect to generate knowledge from this project within 1 month and rapidly contribute to the international response against COVID-19.

- A further strength of this protocol is the strong methodological design that is in accordance with Preferred Reporting Items for Systematic Review and Meta-Analysis Protocols guidelines.

- A possible limitation to the proposed systematic review is the lack of data pertaining to individuals with mental disorders during pandemic or epidemic settings; limited data may prevent us from conducting subgroup meta-analyses, as outlined under the Objectives section.

Systematic review registration PROSPERO registration number: CRD42020179611.

\section{INTRODUCTION}

\section{Rationale}

In December 2019, the scientific community became aware of a novel virus, SARS-CoV-2. This virus and its resultant disease, COVID19 , has led to an international pandemic. As of 26 July 2020, over 16 million people around the globe have been infected with the virus and over 640000 have died. ${ }^{1}$ It is likely that the ramifications of the COVID-19 pandemic will be far reaching; the virus has led to significant disruptions in social connection, economic stability, educational pursuits, 
political decision-making and, most significantly, human health.

Past data suggest that widespread health crises, such as epidemics and pandemics, can spur and compound psychosocial suffering in the general population. Research demonstrates that experiencing physical illnesses and traumatic life events increases the risk of suicidal behaviour. ${ }^{2}$ Studies and reviews of past epidemics demonstrate the potential for the COVID-19 pandemic and its consequences to increase the risk of suicide in non-clinical populations. ${ }^{3-5}$ Indeed, the 2003 SARS epidemic was associated with increased emotional distress and with completed suicides. ${ }^{67}$ Further, during the H1N1 epidemic, the anticipation or reality of oneself or a loved one becoming ill was associated with heightened anxiety. ${ }^{8}$ It is expected that many people will experience increased stress, coping responses to stress (eg, substance use), and perhaps, the onset of mental disorders, as a result of the COVID-19 pandemic. $^{9}$

Governing bodies around the world have rapidly enacted measures to curb the COVID-19 health crisis and prevent deaths. Examples of such measures include social distancing, quarantine, lockdown, mass-media campaigns, non-essential workplace and school closures, as well as transportation shutdowns.

Many of these measures have directly and indirectly been linked to mental health issues and worse mental health outcomes in non-clinical populations. ${ }^{9}$ In a recent review, quarantine was found to be associated with traumatic stress symptoms, depressive symptoms and loneliness. ${ }^{10}$ Social isolation and loneliness are strong risk factors for depressive symptomatology, anxiety and substance use, as well as suicidal ideation and behaviour. ${ }^{211} 12$ Furthermore, non-essential workplace closures during the COVID-19 outbreak have led to record-high rates of job insecurity and unemployment ${ }^{13}$; factors that consistently correlate with depressive symptomatology. ${ }^{14}$

An abundance of research, including a recent systematic review on COVID-19, ${ }^{15}$ has shown that healthcare workers report high rates of mood disturbance and had an increased vulnerability to clinical mental disorders during pandemic settings. In a recent study, Italian physicians who visited a greater number of COVID-19-positive patients were more likely to suffer from symptoms of anxiety and depression than those who visited fewer of these patients. ${ }^{16}$ Interestingly, a recent study found that non-medical personnel (ie, allied health, technicians, maintenance workers) working in hospital settings during a COVID-19 outbreak in Singapore were also at heightened risk for developing mood disorders, anxiety disorders and post-traumatic stress disorder. ${ }^{17}$ Emerging literature certainly suggests that personnel who work in clinical settings during COVID-19 will be at a higher risk for developing mental disorders.

Importantly, the stress of pandemics and epidemics often disproportionately affects marginalised populations. ${ }^{18} 19$ For example, low-income workers facing job insecurity are more likely to experience anxiety during quarantine measures. ${ }^{20}$ Evidence suggests that people with pre-existing mental disorders are more susceptible to the effects of major life stress, including epidemics. A previous study examined the mental health status of 1700 people who were quarantined during the Middle East respiratory syndrome (MERS) Korean outbreak in 2015. After controlling for sex and age, having a premorbid mental disorder increased the likelihood of persistent anxiety and anger at 4-6 months post-quarantine. ${ }^{21}$

The disruption in social support and healthcare services during the COVID-19 pandemic may contribute to worsened outcomes for people with mental disorders. A number of mental health experts have expressed concern over potential ramifications of COVID-19, and succedent measures, for people with pre-existing mental disorders. ${ }^{322}{ }^{23}$ For instance, Yang et al reported that transportation shutdowns will interfere with some patients' ability to access monthly medication that is crucial to their stabilisation. ${ }^{24}$ Further, the MQ: Transforming Mental Health stakeholder survey ${ }^{9}$ collected qualitative data on mental health concerns during the COVID-19 pandemic, and results predicted increased distress in those with premorbid psychiatric disorders. The online survey collected responses from 2200 people between 25 and 27 March 2020; the majority of participants were Caucasian women living in the UK, and $70 \%$ reported having lived experience with mental disorders. Stakeholders were most often concerned about the effects of social distancing, such as the impact of loneliness on people with pre-existing mental disorders. Other prevailing concerns included lack of access to mental health supports and services, exacerbation of pre-existing mental disorders, and increased anxiety and depressive symptomatology. ${ }^{9}$

In addition to the social consequences related to COVID-19, viruses can also induce neurotoxicity and lead to permanent damage to the central nervous system. ${ }^{25} 26$ Although causality is difficult to establish, the 1918 Spanish influenza coincided with a significant increase in the incidence of post-encephalitis parkinsonism. ${ }^{27}$ Preliminary data suggest that one-third of patients hospitalised with COVID-19 have neurological manifestations of disease. ${ }^{18}$ Given the potential for viruses like SARS-CoV-2 to exacerbate pre-existing neuropsychiatric symptoms, individuals with premorbid mental disorders may be disproportionately impacted by pandemics and epidemics through direct, biological effects.

Literature from past health crises suggests that people with psychiatric disorders may be disproportionately affected by the direct and indirect effects of the current COVID-19 pandemic. Thus far, global attention and scientific research have predominantly focused on mental health consequences in patients with COVID-19, frontline healthcare providers and the general population. ${ }^{23}$ Despite disruption to healthcare infrastructure and social supports, the potential neuropsychiatric harms of the SARS-CoV-2 virus and past data indicating heightened vulnerability, there is little published data that report 
on mental health functioning among individuals with pre-existing psychiatric conditions during the current pandemic. ${ }^{9}$ Indeed, the impact of the pandemic on patients with psychiatric disorders may well be different, and potentially more severe, than the impact on the general population. ${ }^{21}$ The current body of literature is lacking with respect to how psychiatric patients are functioning during COVID-19; the answer to which we hope will be partially elucidated by our review. To do so, we will review and synthesise data from past epidemics/ pandemics, as well as the present pandemic, that report on mental health outcomes in patients with psychiatric disorders that predate the onset of the epidemic/pandemic. Knowledge generated from our systematic review will also assess the state of evidence on the neuropsychiatric symptoms of COVID-19 in these patients. Thus, we will identify gaps and findings that are transferrable to the current pandemic, as well as inform healthcare services, patients and society on how to best support patients with mental disorders during epidemics/pandemics. We seek to answer the following: For people with pre-existing mental disorders, what is the impact of pandemics and epidemics on mental health outcomes? What are the neuropsychiatric manifestations of COVID-19 in these patients? What has been beneficial in supporting patients with mental illness during past health crises?

\section{Objectives}

Our primary objective seeks to determine the impact of pandemics and epidemics on the mental health of people with pre-existing mental disorders. The majority of recent reviews published on COVID-19 and psychiatric outcomes report on the psychiatric and/or neuropsychiatric effects of COVID-19 in the general population. ${ }^{28} 29$ To our knowledge, only one review (as of 19 July 2020) specifically examines psychiatric populations in this context; however, this review only examined persons with psychosis and excluded participants with other mental disorders. ${ }^{30}$ Further, the previously mentioned review included studies reporting on patients whose psychosis presented during an epidemic/pandemic. In contrast, our planned review will explore the effects of pandemics or epidemics on individuals with any mental disorder, not limited to individuals affected by psychosis, and will not report on those with disorders diagnosed during, or as a result of, epidemics/pandemics. As research regarding the effects of COVID-19 on individuals with pre-existing psychiatric diagnoses continues to be produced, ${ }^{31} 32$ we hope that our findings will provide an up-to-date synthesis on this specific body of literature, with an ultimate goal of improving care for this vulnerable sector of the population during this international pandemic. In this systematic review, we aim to synthesise existing literature on the impact of pandemics and epidemics on people with premorbid mental disorders. We will also conduct subgroup analyses if an adequate number of studies are found for the group of interest. ${ }^{33}$ Most subgroups are exploratory; however, some were selected based on previous literature. Whenever possible, we have provided a hypothesis. Proposed subgroup analyses are as follows:

1. Type of mental disorder.

2. Identity factors (age, sex, gender, ethnicity, sexual orientation): We hypothesise that traditionally marginalised and vulnerable groups in society (ie, young people ( $<25$ years), seniors, women, females, ethnic minorities, LGBTQ+ community members) with mental disorders will experience worse mental health outcomes during pandemics and epidemics. Research demonstrates that disadvantaged and oppressed groups are more likely to suffer from the physical and mental ramifications of a previous influenza outbreak. ${ }^{34}$

3. Region.

4. Specific pandemic (eg, COVID-19).

5. Specific epidemic.

6. Specific measures to curb pandemics/epidemics (eg, quarantine): We hypothesise that measures grounded in social distancing (eg, quarantine, legally mandated isolation) will show the strongest association with adverse psychiatric outcomes in people with pre-existing mental disorders. While data on those with pre-existing mental disorders may be scant, data from the general population suggest that longer quarantine duration and isolation measures that impede access to resources can worsen psychological outcomes, which may also apply to this specific population. ${ }^{10}$ As mentioned previously, social isolation is also a strong predictor of a number of psychiatric diseases. ${ }^{211} 12$

We have two additional objectives as follows:

Secondary objective: Assimilate and examine the current evidence for neuropsychiatric manifestations of SARS-CoV-2 and the impact on patients with pre-existing mental disorders. For this particular objective, we will only report on data from the COVID-19 pandemic and will exclude historical epidemics/pandemics, in order to synthesise the growing body of literature on the specific neuropsychiatric manifestations of this novel virus and aid the global scientific community. In order to do so, we have incorporated search terms describing neuropsychiatric presentations such as 'confusion',' 'delirium', but, in keeping with a recent review, ${ }^{29}$ will exclude terms of presentations such as 'encephalitis', 'stroke' and 'seizure' that can exist without psychiatric sequelae or manifestation. We will present these data in table format.

Tertiary objective: With input from knowledge users and people with lived experience, summarise relevant mental health resources that can be used during the current pandemic while maintaining physical distancing.

Our three objectives meet a number of the recommendations and calls to actions as outlined by Holmes et al. ${ }^{9}$

\section{METHODS}

\section{Eligibility criteria}

Types of studies

We will include both observational and experimental studies as well as qualitative reports, case studies or series, 
case-control studies, longitudinal studies and cohort studies. We will also gather information from alternative sources, such as letters to editors, correspondences and opinions, as well as from people with lived experience directly. Only reports written in English will be included.

\section{Types of participants}

Our population of interest is people with mental disorders whose diagnosis predated the onset of a pandemic or epidemic. Studies exploring mental health outcomes in the general population, or mental disorders diagnosed during a pandemic or epidemic, will not be included. We will not exclude studies based on demographics; studies reporting on every sex, gender, ethnicity and age are relevant.

\section{Types of exposures}

We will include articles pertaining to any pandemic or epidemic throughout history, including 'Spanish influenza' (H1N1), 'Asian influenza' (H2N2), AIDS (HIV), 'Swine influenza' (H1N1), 'SARS' (SARS-CoV), 'MERS' (MERS-CoV) and 'COVID-19' (SARS-CoV-2). We expect the time frame for publication could range from the 1918 Spanish influenza pandemic (considered to be the first pandemic in modern society) up until present day (ie, COVID-19 pandemic).$^{35}$ We will align our inclusion criteria for an epidemic with the WHO's definition: an 'occurrence in a community or region of cases of an illness, specific health-related behaviour, or other healthrelated events clearly in excess of normal expectancy. ${ }^{, 36}$ Pandemics will also be defined based on the WHO definition: 'an epidemic occurring worldwide, or over a very wide area, crossing international boundaries and usually affecting a large number or people' and in which 'almost simultaneous transmission occurs worldwide. ${ }^{37}$ Certain past epidemics or pandemics included in this review, such as HIV/AIDS, may differ from the COVID-19 pandemic in terms of their virus profile, mode of transmission, time course and resultant public health strategies. ${ }^{30}$ In the likely case that we identify an adequate number of articles reporting on specific diseases and their impact on people with pre-existing mental disorders, such as HIV/AIDS, we will conduct subgroup analyses (eg, by pathogen, by mode of transmission) and report on these data separately.

Given the challenge of separating the effect of the pandemic or epidemic itself from measures employed during these crises, we will also include studies reporting on the following exposures: all forms of social distancing, physical distancing, travel suspensions, mass-media campaigns, as well as closures of schools, non-essential workplaces, and mental health services/treatment facilities, if enacted due to a pandemic or epidemic.

\section{Outcomes}

We will examine the following mental health outcomes: anxiety and depressive symptomatology, substance use, relapse rate, suicidal behaviour (suicidal ideation, behaviour, attempts, as well as completed suicide rate), psychiatric emergency room visits, psychiatric hospitalisation, crisis intervention uptake and mental health-related family physician visits. Our comparison is this population's pre-exposure stability, severity of symptomatology and access to care. We expect studies to employ a variety of different scales to report symptomatology, substance use, and suicidal ideation and behaviour, such as the Beck Depression Inventory or Alcohol Use Disorders Identification Test. We expect that other studies could report on a quantitative change in service utilisation, relapse rate and suicide rate, for the remaining outcomes of interest. In the reporting of our outcomes, results from non-standardised tools will be described qualitatively as these studies cannot identify outcomes with any degree of certainty.

Our secondary objective is to synthesise the literature on the neuropsychiatric manifestations of COVID-19 in psychiatric patients. For this objective, we will only report on data from the COVID-19 pandemic and will not consider data reporting on other pathogens or pandemics. The rationale for this decision is outlined here. First, elucidating and summarising information specifically about how the novel SARS-CoV-2 can present neuropsychiatrically in people with pre-existing mental disorders, and also exacerbate these pre-existing conditions, is exceptionally important to patient care during the pandemic. Indeed, the neuropsychiatric effects of this virus may be different from other viruses or pathogens from a pathophysiological perspective. Furthermore, the neuropsychiatric manifestations of pathogens are a generally understudied topic, and as such, inclusion of similar data from previous epidemics/pandemics may muddle these analyses. ${ }^{38}$

In order to resolve our tertiary objective, we will also explore measures that have been beneficial in supporting patients with mental disorders during past health crises. These resources will be identified from the included studies as well as through a specific search to identify virtual healthcare tools.

\section{Outcomes and prioritisation}

The primary outcomes are as follows: anxiety and depressive symptomatology, substance use, relapse rate (a change in mental status), suicidal behaviour, psychiatric emergency room visits, psychiatric hospitalisation, crisis intervention uptake and mental health-related family physician visits. Our secondary outcomes are neuropsychiatric signs and symptoms of COVID-19, including, but not limited to confusion, apathy and delirium. Our third outcome is mental health resources that were successfully employed during past pandemics and epidemics to support psychiatric patients.

\section{Information sources}

Studies were collected for this systematic review through a carefully laid-out search strategy developed in collaboration with research experts and a health sciences librarian (SS), who is experienced in systematic review data 
searches. The search strategy was tested in a single database and then adapted to our other databases of interest. The following databases were searched: MEDLINE/ PubMed, CINAHL, PsycINFO, medRxiv and Google Scholar. The initial search was conducted on 5 May 2020. A second search was conducted 23 July 2020, after the systematic review protocol was finalised and peer reviewer comments had been incorporated into the search strategy. Publications were identified by a combination of search terms relating to mental disorders and pandemics (refer to table 1). In addition to the initial database search, the study team will manually review the references of all relevant articles to ensure that no pertinent articles were missed. The date for the literature search will be set from 1918, the first documented pandemic, ${ }^{24}$ until 7 May 2020 (date of article submission).

\section{Search strategy}

See table 1.

\section{Study records}

\section{Data Management}

Relevant articles identified in the database search and reference evaluations will be uploaded on to the Covidence $^{39}$ platform for systematic reviews. This platform will enable the study team to compile articles and flag any redundancy in the search results.

\section{Selection process}

The selection process will involve two independent reviewers who will conduct the initial title and abstract screen, followed by a full text extraction, in duplicate. If any disagreements between authors occur during these processes, a discussion will ensue in an attempt to reach consensus. If consensus cannot be achieved, a third reviewer will be consulted. Once eligibility has been established, the two independent reviewers will determine which articles should be included for specific metaanalyses (if feasible) in a format akin to that described above.

\section{Data extraction/abstraction}

Data will be collected by two independent reviewers using the Covidence platform for systematic reviews. Data extraction forms will be created and completed within this software program. Refer to the Data items section for information regarding the type of data that will be collected for the purpose of this review.

\section{Data items}

We will extract article reference details from all eligible studies, including author names and titles, publication title, journal or site of publication, volume and issue (if applicable), year published, country of publication and conflict of interest statements. Reviewers will also collect content-related data for each article, including study methodology and design, population demographics and sample size, mental disorder diagnosis, symptomatology measure (if applicable), study outcomes and findings, and implications.

\section{Risk of bias}

As this study will include both observational and randomised controlled trial data, two scales will be used to determine risk of bias. The Newcastle Ottawa Scales will be used to evaluate the observational studies included in the review. This scale assesses the quality of data in a starsystem that judges a publication based on three categories: 'the selection of the study groups; the comparability of the groups; and the ascertainment of either the exposure or outcome of interest for case-control or cohort studies respectively. ${ }^{40}$ Based on the number of stars received for each section, papers are graded as (1) good quality, (2) fair quality or (3) poor quality. The Cochrane Collaboration's Tool will be used to assess the included randomised controlled trials. The Cochrane scale covers six domains of bias: selection bias, performance bias, detection bias, attrition bias, reporting bias, and other bias, and each domain is evaluated in a point-based system. ${ }^{41}$ This tool produces a grading of the evidence for each paper in the review as follows: low risk of bias, unclear risk of bias or high risk of bias. We will present the risk of bias for each included study in table format. Case studies, commentaries and qualitative papers included in our review will not be assessed for quality with standardised tools.

\section{Data synthesis}

We expect our data comparisons to be challenged by differing methodological approaches employed between papers, especially given the possibility of identifying historical data. As such, we will be guided by the following plan:

1. Results will be reported narratively. We will describe study findings individually as well as by specific pandemic and epidemic. We will also construct tables, one for each pandemic or epidemic, that include all papers that reported on psychiatric outcomes in patients with pre-existing mental disorders. It will be noted qualitatively if we find insufficient papers reporting on a given pandemic or epidemic to allow for subgroup analysis and presentation of data within a table format. We will construct a separate table for the reporting of neuropsychiatric manifestations in COVID-19 in these patients. We will also create an easily accessible list of virtual mental health resources that can be used immediately by patients and healthcare providers during the current pandemic.

2. If we identify sufficiently homogeneous studies in terms of clinical and methodological characteristics, we will pool randomised controlled trials and observational studies separately, in order to conduct metaanalyses. In order to conduct meta-analyses, the following conditions must be met: (1) we identify five or greater studies with comparable mental health outcomes; and (2) the majority of included studies are deemed of low risk of bias. In this case, we will report 
Table 1 Database search strategy

\begin{tabular}{|c|c|}
\hline Database & Search strategy \\
\hline $\begin{array}{l}\text { MEDLINE Search } \\
\text { A: } 8060 \\
\text { Search B: } 3596\end{array}$ & 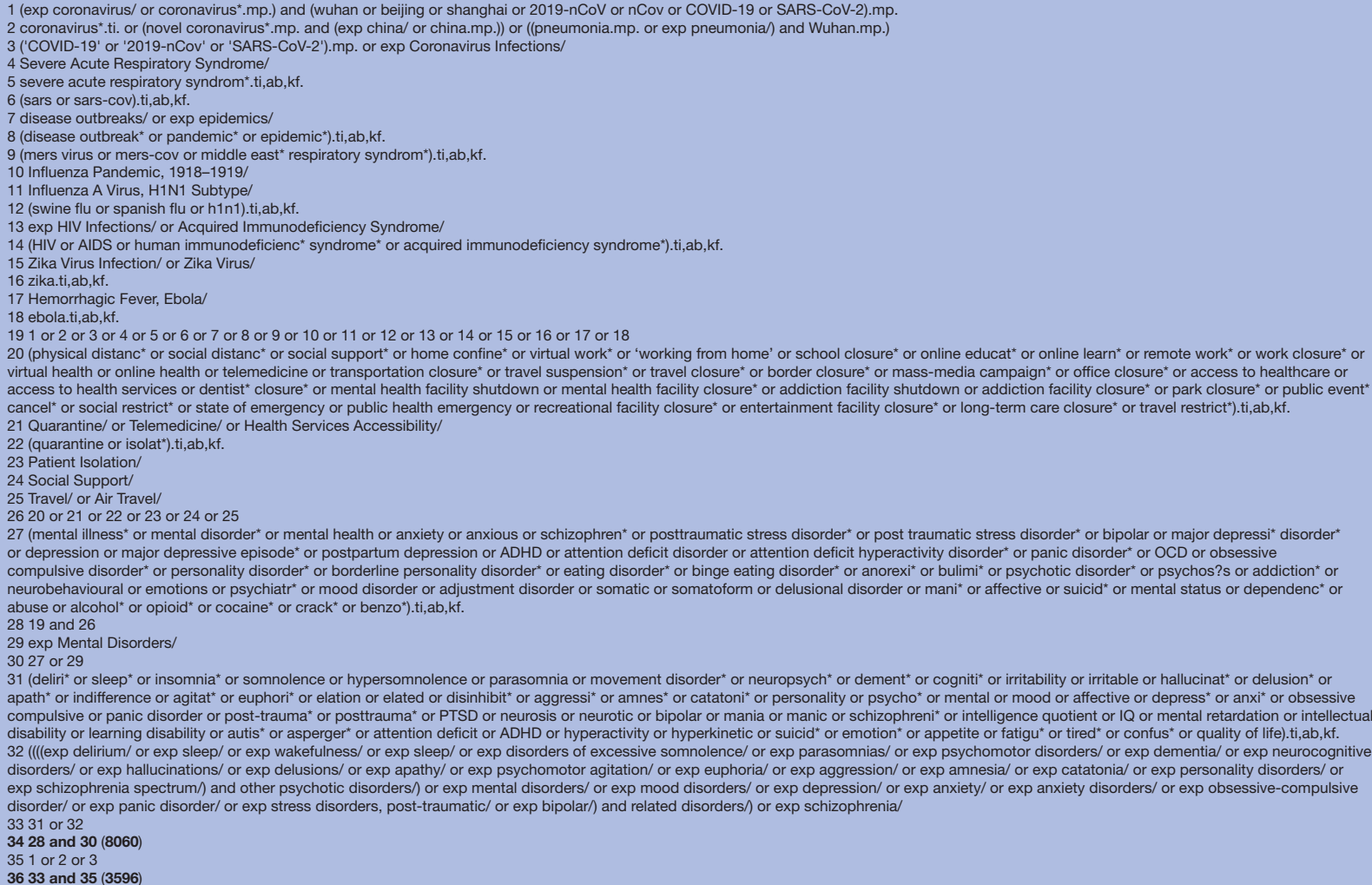 \\
\hline
\end{tabular}

30 (mental illness ${ }^{*}$ or mental disorder* or mental health or anxiety or anxious or schizophren* or posttraumatic stress disorder* ${ }^{*}$ or post traumatic stress disorder* or bipolar or major depressi* disorder* or depression or major depressive episode* or postpartum depression or ADHD or attention deficit disorder or attention deficit hyperactivity disorder* or panic disorder" or borderline personality disorder ${ }^{*}$ or OCD or obsessive compulsive disorder* or personality disordes* or borderline personality disorder* or eating disorder* or binge eating disorder* or anorexi* or bulimi* or psychotic disorder or $^{*}$ or psychos?
or addiction* dependenc* or abuse or alcohol* or opioid* or cocaine ${ }^{*}$ or crack $^{*}$ or benzo*).ti,ab. 3129 or 30

3228 and $31(2365)$

33 (deliri apath* or indifference or agitat ${ }^{*}$ or euphorit or elation or elated or disinhibit* or aggressi" or amnes* or catatoni* or personality or psycho* or mental or mood or affective or depress ${ }^{*}$ or anxi* or obsessive compulsive or panic disorder or post-trauma* or posttrauma* or PTSD or neurosis or neurotic or bipolar or mania or manic or schizophreni* or intelligence quotient or IQ or mental retardation or intellectual disability or learning disability or autis* or asperger" or attention deficit or ADHD or hyperactivity or hyperkinetic or suicid* or emotion* or appetite or fatigu* or tired ${ }^{*}$ or confus* or quality of life).ti,ab. 34 (((lexp delirium/ or exp sleep/ or exp wakefulness/ or exp sleep/or exp disorders of excessive somnolence/ or exp parasomnias/ or exp psychomotor disorders/or exp dementia/ or exp neurocognitive disorders/ or exp hallucinations/ or exp delusions/ or exp apathy/ or exp psychomotor agitation/or exp euphoria/ or exp aggression/or exp amnesia/ or exp catatonia/ or exp personality disorders/ or exp schizophrenia spectrum// and other psychotic disorders/) or exp mental disorders/ or exp mood disorders/ or exp depression/ or exp anxiety/ or exp anxiety disorders/ or exp obsessive-compulsive disorder/ or exp panic disorder/ or exp stress disorders, post-traumatic/ or exp bipolar/) and related disorders/) or exp schizophrenia/ 3533 or 34 361 or 2 or 3

3735 and $36(514)$ 
Table 1 Continued

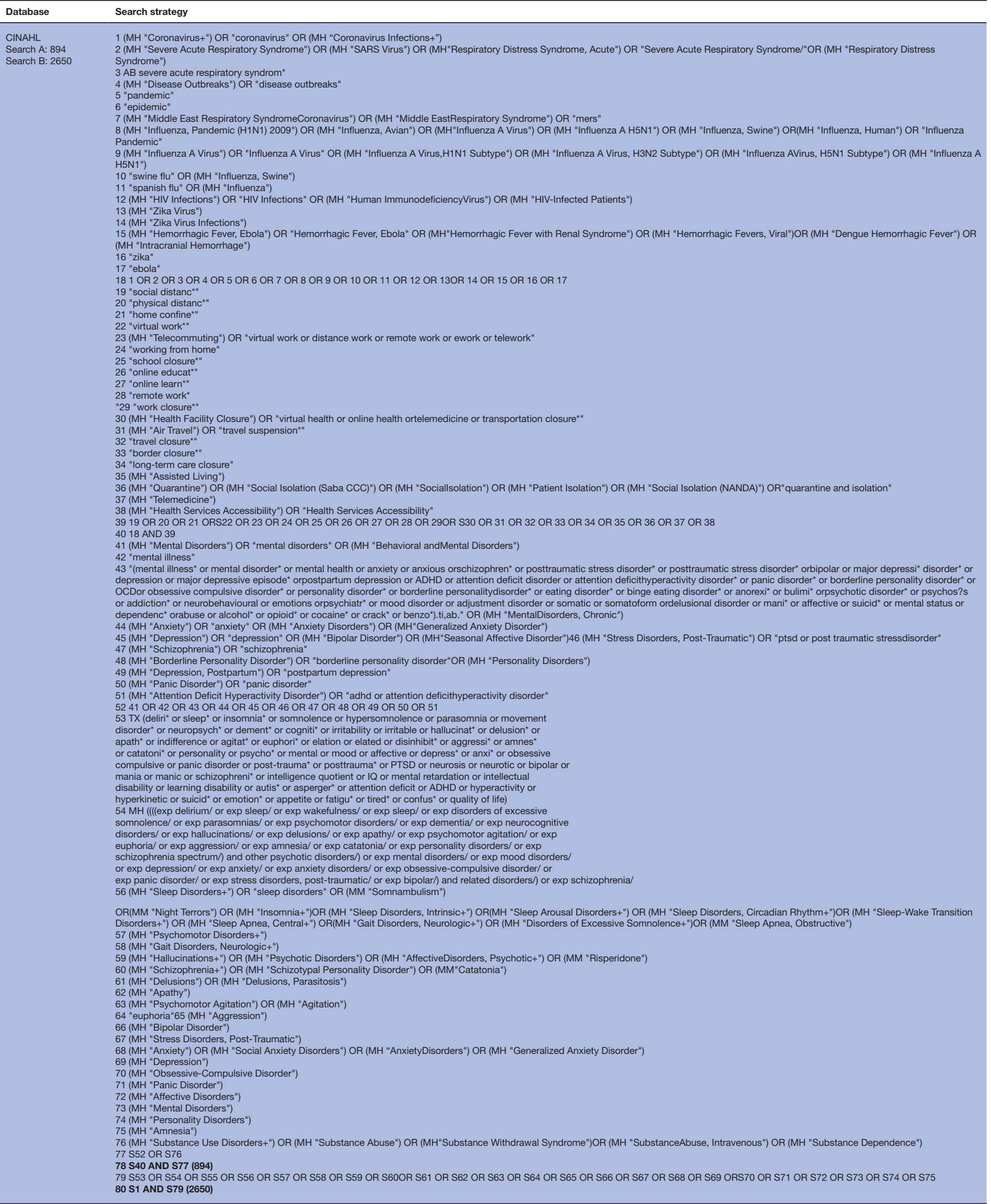




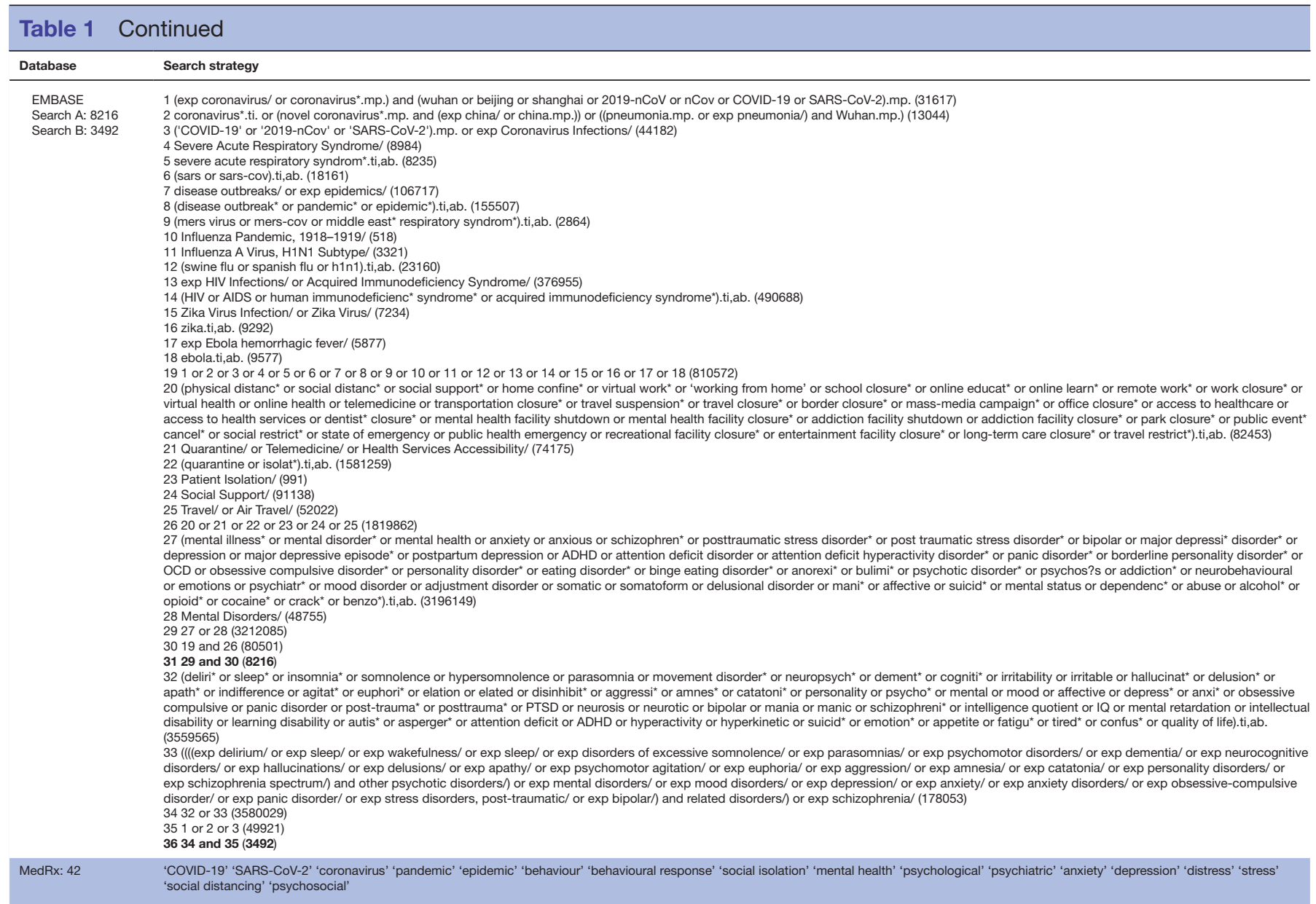

aggregated effect sizes. Standardised mean differences will be employed for continuous variables and ORs for dichotomous variables.

3. In the case of substantive data that meet the criteria outlined above, we will proceed with subgroup analyses. If possible, we will examine outcomes by mental health disorder, by identity factors (such as age, sex, gender), by country, by specific pandemic and epidemic, and by distinct measures used to curb pandemics and epidemics (eg, quarantine).

4. We will use a metasynthesis approach for the synthesis of qualitative literature.

We will use the software RevMan V.5. $3^{42}$ to conduct meta-analyses.

\section{Meta-bias}

If at least 10 studies are included in the systematic review, and an effect size is created from meta-analysis, an Egger's plot will be performed in order to assess for publication bias. This method will ensure that results are not biassed toward one outcome over another.

\section{Confidence in the cumulative evidence}

The quality of the evidence will be evaluated using the Grading of Recommendations, Assessment, Development, and Evaluation (GRADE) system if there are enough studies in the review to undertake a meta-analysis.
The GRADE framework allows authors to grade papers based on risk of bias, imprecision, inconsistency, indirectness and publication bias. ${ }^{43}$ GRADE ratings are reported as follows: (1) very low certainty; (2) low certainty; (3) moderate certainty; and (4) high certainty. The certainty grading for each paper can then be used to ascertain the confidence in the evidence as a whole. By uploading the meta-analyses files into the GRADE system, we will be able to report on the confidence of our findings.

\section{Presenting and reporting of results}

The present protocol document is written in accordance with the Preferred Reporting Items for Systematic Review and Meta-Analysis Protocols (PRISMA-P) guidelines for systematic reviews. ${ }^{44}$ The final paper will also adhere to these guidelines.

\section{Patient and public involvement}

Our team collaborated with a patient with recent lived experience with mental illness and suicidality throughout the conception of this project. This team member was instrumental in designing the primary research question in particular. We have arranged for regular meetings with this patient team member and will continue to have her input moving forward. We expect that this will be especially helpful during the creation of our list of mental health resources that can be used during the COVID-19 
pandemic by patients and mental health providers. This team member will also assist in the dissemination process, by editing the final manuscript as well as informing creation of educational sessions for medical students and psychiatry residents.

\section{DISCUSSION}

To our knowledge, this will be the first study to synthesise the state of evidence on the impact of pandemics and epidemics on psychiatric patients. We believe that this will also be the first project to summarise the neuropsychiatric manifestations of COVID-19. Through rigorous screening and synthesising of the literature, we hope that our systematic review will shed light on how health crises, including the current COVID-19 pandemic, impact individuals with pre-existing mental disorders.

The effects of the COVID-19 pandemic are expected to intensify over the coming weeks and months. ${ }^{45}$ Given the potential for disastrous consequences to psychiatric patients, due to social consequences and neuropsychiatric manifestations of disease, and the gap in the literature that presently exists, rapid and collaborative research on psychiatric populations during COVID-19 is imperative. In order to contribute to the literature and fulfil recently published calls for action, ${ }^{9}$ our research collaborative will conduct a large-scale retrospective chart analysis of psychiatric patients in Canada following this systematic review.

By conceptualising the existing literature, the present systematic review will lay the groundwork for our future research. We hope that our review will also encourage other scientists to research this vulnerable population during the COVID-19 pandemic. Our goal is to produce the high-quality evidence necessary to inform future innovations and policy changes that can effectively support people with mental disorders during and after this pandemic.

In the interim, widespread education and implementation of services for individuals with mental disorders is crucial in order to prevent undue suffering. As such, and in collaboration with people with lived experience, we will publicise a list of mental health resources that can be used by clinicians and by patients, immediately. By synthesising epidemiological data on neuropsychiatric signs and symptoms of COVID-19, we also hope to assist the global scientific community in characterising this novel virus. We anticipate that this review will make substantial contributions to future researchers, policy makers, clinicians, people with mental disorders and overarchingly, to the national and international response to COVID-19.

In the meantime, we strongly advocate for the urgent implementation of support services for individuals with mental disorders. As a result of 'non-essential closures', people with mental disorders around the world have been left without access to crucial treatment services. While innovation of evidenced-based interventions is important, it is also critical that provisional supports be made available immediately.

\section{ETHICS AND DISSEMINATION}

No ethical approval is required for this protocol and proposed systematic review as we will only use data from previously published papers that have themselves received ethics clearance and used proper informed consent procedures. The results of our review will be published in a peer-reviewed journal.

\section{Author affiliations}

${ }^{1}$ Undergraduate Medical Education Program, Michael G. DeGroote School of Medicine, McMaster University Faculty of Health Sciences, Hamilton, Ontario, Canada

${ }^{2}$ Department of Psychiatry and Behavioural Neurosciences, McMaster University Faculty of Health Sciences, Hamilton, Ontario, Canada

${ }^{3}$ Medical Science Graduate Program, McMaster University, Hamilton, Ontario, Canada

${ }^{4}$ Health Sciences Library, McMaster University, Hamilton, Ontario, Canada ${ }^{5}$ Community member, Hamilton, Ontario, Canada

${ }^{6}$ Department of Health Research, Evidence and Impact, McMaster University, Hamilton, Ontario, Canada

\section{Twitter Emma Alaine van Reekum @EmmavanReekum}

Contributors All authors were involved in revising the manuscript and approved the final version for publication. Each author made substantial contributions to the conception of the work or analytic plan. EAvR, AS and ZS conceived the initial research questions. NS, SL, SS, LM and LT contributed to the study design and analytic plan. AS and EAvR drafted the manuscript and made the requested revisions. TR, AD and ZS critically revised the manuscript. All authors have approved the submitted version and agree to be personally accountable for the integrity and accuracy of the work.

Funding The authors have not declared a specific grant for this research from any funding agency in the public, commercial or not-for-profit sectors.

Competing interests None declared.

Patient and public involvement Patients and/or the public were involved in the design, or conduct, or reporting, or dissemination plans of this research. Refer to the Methods section for further details.

Patient consent for publication Not required.

Provenance and peer review Not commissioned; externally peer reviewed.

Open access This is an open access article distributed in accordance with the Creative Commons Attribution Non Commercial (CC BY-NC 4.0) license, which permits others to distribute, remix, adapt, build upon this work non-commercially, and license their derivative works on different terms, provided the original work is properly cited, appropriate credit is given, any changes made indicated, and the use is non-commercial. See: http://creativecommons.org/licenses/by-nc/4.0/.

\section{ORCID iDs}

Anjali Sergeant http://orcid.org/0000-0002-2076-8901

Nitika Sanger http://orcid.org/0000-0002-5883-1873

Tea Rosic http://orcid.org/0000-0001-7406-4056

Lawrence Mbuagbaw http://orcid.org/0000-0001-5855-5461

Zainab Samaan http://orcid.org/0000-0002-5974-9361

\section{REFERENCES}

1 Our World in Data. "Coronavirus Pandemic (COVID-19).", 2020. Available: https://ourworldindata.org/coronavirus [Accessed $26 \mathrm{Jul}$ 2020].

2 O'Connor RC, Nock MK. The psychology of suicidal behaviour. Lancet Psychiatry 2014;1:73-85.

3 Gunnell D, Appleby L, Arensman E, et al. Suicide risk and prevention during the COVID-19 pandemic. Lancet Psychiatry 2020;7:468-71. 
4 Cheung YT, Chau PH, Yip PSF. A revisit on older adults suicides and severe acute respiratory syndrome (SARS) epidemic in Hong Kong. Int J Geriatr Psychiatry 2008;23:1231-8.

5 Wasserman IM. The impact of epidemic, war, prohibition and media on suicide: United States, 1910-1920. Suicide Life Threat Behav 1992;22:240-54.

6 Nickell LA, Crighton EJ, Tracy CS, et al. Psychosocial effects of SARS on hospital staff: survey of a large tertiary care institution. CMAJ 2004;170:793-8.

7 Chan SMS, Chiu FKH, Lam CWL, et al. Elderly suicide and the 2003 SARS epidemic in Hong Kong. Int J Geriatr Psychiatry 2006;21:113-8.

$8 \mathrm{Xu} \mathrm{J}$, Zheng Y, Wang M, et al. Predictors of symptoms of posttraumatic stress in Chinese university students during the 2009 H1N1 influenza pandemic. Med Sci Monit 2011;17:PH60-4.

9 Holmes EA, O'Connor RC, Perry VH, et al. Multidisciplinary research priorities for the COVID-19 pandemic: a call for action for mental health science. Lancet Psychiatry 2020;7:547-60.

10 Brooks SK, Webster RK, Smith LE, et al. The psychological impact of quarantine and how to reduce it: rapid review of the evidence. The Lancet 2020;395:912-20.

11 Cacioppo JT, Hughes ME, Waite LJ, et al. Loneliness as a specific risk factor for depressive symptoms: cross-sectional and longitudinal analyses. Psychol Aging 2006;21:140-51.

12 Matthews T, Danese A, Caspi A, et al. Lonely young adults in modern Britain: findings from an epidemiological cohort study. Psychol Med 2019;49:268-77

13 Woolhandler S, Himmelstein DU, Intersecting US. Intersecting U.S epidemics: COVID-19 and lack of health insurance. Ann Intern Med

14 Dooley D, Prause J, Ham-Rowbottom KA. Underemployment and depression: Iongitudinal relationships. J Health Soc Behav 2000;41:421-36.

15 Pappa S, Ntella V, Giannakas T, et al. Prevalence of depression, anxiety, and insomnia among healthcare workers during the COVID-19 pandemic: a systematic review and meta-analysis. Brain Behav Immun 2020;88:901-7.

16 Amerio A, Bianchi D, Santi F, et al. Covid-19 pandemic impact on mental health: a web-based cross-sectional survey on a sample of Italian general practitioners. Acta Biomed 2020;91:83-8.

17 Tan BYQ, Chew NWS, Lee GKH, et al. Psychological impact of the COVID-19 pandemic on health care workers in Singapore. Ann Intern Med 2020:M20-1083.

18 Douglas PK, Douglas DB, Harrigan DC, et al. Preparing for pandemic influenza and its aftermath: mental health issues considered. Int J Emerg Ment Health 2009;11:1-8.

19 Rothstein MA. From SARS to Ebola: legal and ethical considerations for modern quarantine. Indiana Health Law Rev 2015;12:227-80.

20 Blake KD, Blendon RJ, Viswanath K. Employment and compliance with pandemic influenza mitigation recommendations. Emerg Infect Dis 2010:16:212-8.

21 Jeong $\mathrm{H}$, Yim HW, Song Y-J, et al. Mental health status of people isolated due to middle East respiratory syndrome. Epidemiol Health 2016;38:e2016048:1-7.

22 Druss BG. Addressing the COVID-19 pandemic in populations with serious mental illness. JAMA Psychiatry 2020:jamapsychiatry.2020.0894.

$23 \mathrm{Yao} \mathrm{H}$, Chen J-H, Xu Y-F. Patients with mental health disorders in the COVID-19 epidemic. Lancet Psychiatry 2020;7:e21.

24 Yang Y, Li W, Zhang Q, et al. Mental health services for older adults in China during the COVID-19 outbreak. Lancet Psychiatry 2020;7:e19.

25 Swanson PA, McGavern DB. Viral diseases of the central nervous system. Curr Opin Virol 2015;11:44-54.
26 Mao L, Jin H, Wang M, et al. Neurologic manifestations of hospitalized patients with coronavirus disease 2019 in Wuhan, China JAMA Neurol 2020:1127.

27 McCall S, Vilensky JA, Gilman S, et al. The relationship between encephalitis lethargica and influenza: a critical analysis. J Neurovirol 2008;14:177-85

28 Rajkumar RP. COVID-19 and mental health: a review of the existing literature. Asian J Psychiatr 2020;52:102066.

29 Rogers JP, Chesney E, Oliver D, et al. Psychiatric and neuropsychiatric presentations associated with severe coronavirus infections: a systematic review and meta-analysis with comparison to the COVID-19 pandemic. Lancet Psychiatry 2020;7:611-27.

30 Brown E, Gray R, Lo Monaco S, et al. The potential impact of COVID-19 on psychosis: a rapid review of contemporary epidemic and pandemic research. Schizophr Res 2020:j.schres.2020.05.005.

31 Davide P, Andrea P, Martina O, et al. The impact of the COVID-19 pandemic on patients with OCD: effects of contamination symptoms and remission state before the quarantine in a preliminary naturalistic study. Psychiatry Res 2020;291:113213.

32 Fischer M, Coogan AN, Faltraco F, et al. COVID-19 paranoia in a patient suffering from schizophrenic psychosis - a case report. Psychiatry Res 2020;288:113001.

33 Cochrane. "What are subgroup analyses". Available: https:// handbook-5-1.cochrane.org/chapter_9/9_6_2_what_are_subgroup_ analyses.htm [Accessed $26 \mathrm{Jul}$ 2020].

34 Quinn SC, Kumar S. Health inequalities and infectious disease epidemics: a challenge for global health security. Biosecur Bioterror 2014;12:263-73.

35 Tomes N. "Destroyer and teacher": Managing the masses during the 1918-1919 influenza pandemic. Public Health Rep 2010;125 Suppl 3:48-62.

36 World Health Organization. "Definitions: Emergencies”, 2014. Available: https://who.int/hac/about/definitions/en/ [Accessed $17 \mathrm{Apr}$ 2020]

37 Kelly $\mathrm{H}$. The classical definition of a pandemic is not elusive. Bull World Health Organ 2011;89:540-1.

38 Troyer EA, Kohn JN, Hong S. Are we facing a crashing wave of neuropsychiatric sequelae of COVID-19? neuropsychiatric symptoms and potential immunologic mechanisms. Brain Behav Immun 2020;87:34-9.

39 Covidence. Covidence systematic review software, Veritas Health Innovation,Melbourne, Australia. Available: www.covidence.org

40 Peterson J, Welch V, Losos M, et al. The Newcastle-Ottawa scale (NOS) for assessing the quality of nonrandomised studies in metaanalyses. Ottawa: Ottawa Hospital Research Institute, 2011.

41 Higgins JPT, Altman DG, Gøtzsche PC, et al. The Cochrane collaboration's tool for assessing risk of bias in randomised trials. BMJ 2011;343:d5928.

42 Cochrane RevMan. Review Manager(RevMan) [Computer program] Version 5.3. Copenhagen: The Nordic Cochrane Centre, The Cochrane Collaboration, 2014.

43 Schünemann H, Guyatt G. Criteria for applying or using grade., 2020 Available: https://www.gradeworkinggroup.org/ [Accessed $12 \mathrm{Apr}$ 2020].

44 Moher D, Shamseer L, Clarke M, et al. Preferred reporting items for systematic review and meta-analysis protocols (PRISMA-P) 2015 statement. Syst Rev 2015;4:1-9.

45 Anderson RM, Heesterbeek H, Klinkenberg D, et al. How will countrybased mitigation measures influence the course of the COVID-19 epidemic? Lancet 2020;395:931-4. 